\title{
Off-Diagonal Heat Kernel Asymptotics of Pseudodifferential Operators on Closed Manifolds and Subordinate Brownian Motion
}

\author{
M. A. Fahrenwaldt
}

\begin{abstract}
We derive the off-diagonal short-time asymptotics of the heat kernels of functions of generalised Laplacians on a closed manifold. As an intermediate step we give an explicit asymptotic series for the kernels of the complex powers of generalised Laplacians. Each asymptotic series is formulated in terms of the geodesic distance. The key application concerns upper bounds for the transition density of subordinate Brownian motion. The approach is highly explicit and tractable.
\end{abstract}

Mathematics Subject Classification. Primary 35K08; Secondary 58J65, $35 \mathrm{~S} 05$.

Keywords. Pseudodifferential operators, Complex powers, Heat kernel, Mellin transform, Subordinate Brownian motion.

\section{Introduction}

Let $M$ be a closed Riemannian manifold of dimension $n$ and let $A$ be a generalised Laplacian acting on smooth functions on $M$, i.e. $A$ is a second-order differential operator whose principal symbol is the metric tensor. Given the short-time asymptotics of the heat kernel $K\left(e^{-t A} ; x, y\right)$ of $A$ we represent the off-diagonal kernel of the complex powers $K\left(A^{-z} ; x, y\right)$ as an asymptotic series in powers of the geodesic distance $d(x, y)$. We finally consider the operator $f(A)$ for suitable functions $f$ and give short-time asymptotics of the heat kernel of $f(A)$ denoted by $K\left(e^{-f(A) t} ; x, y\right)$ and Aronson-type upper bounds. Schematically, this can be illustrated as follows where $d$ denotes the geodesic distance on $M$. 


$$
\begin{aligned}
K\left(e^{-t A} ; x, y\right) & \sim\left(\frac{1}{2 \pi t}\right)^{n / 2} e^{-d(x, y)^{2} / 4 t} \sum_{k=0}^{\infty} H_{k}(x, y) t^{k} \text { as } t \rightarrow 0 \\
\Downarrow & \sum_{k=0}^{\infty} \frac{\Phi_{k}(z ; x, y)}{\left(\frac{1}{4} d(x, y)^{2}\right)^{\frac{n}{2}-k-z}}+\sum_{k=0}^{\infty} \Psi_{k}(z ; x, y)\left(\frac{1}{4} d(x, y)^{2}\right)^{k} \\
\Gamma(z) K\left(A^{-z} ; x, y\right) & \Downarrow \\
K\left(e^{-t f(A)} ; x, y\right) & \sim t e^{-m t}\left[H(x, y)+O\left(d(x, y)^{-n-2 \alpha+4}\right)\right] \\
H(x, y) & =\frac{c_{0} H_{0}(x, y)}{d(x, y)^{n+2 \alpha}}+\frac{c_{1} H_{0}(x, y)+c_{2} H_{1}(x, y)}{d(x, y)^{n+2 \alpha-2}} \\
\Downarrow & \quad C t e^{-m t}\left(\left(d(x, y)+t^{1 / 2 \alpha}\right)^{-n-2 \alpha} \wedge H(x, y)\right)
\end{aligned}
$$

for a constants $\alpha$ and $m$ depending on $f$, constants $c_{0}, c_{1}, c_{2}$ and functions $\Phi_{k}, \Psi_{k}$ that depend on the $H_{0}, H_{1}, \ldots$ and will be made explicit.

We obtain these results by the well-known Handelsman-Lew method [6] based on the Mellin transform and complex analysis. This allows an efficient, tractable and explicit computation of the above series to arbitrary order avoiding local calculations on the manifold in terms of operator symbols.

The kernels of the complex powers are not only the key building block in the heat kernel analysis, they are also of independent interest. The ondiagonal behaviour of the kernels is well-understood from index theory, while the above series representation gives a complete description of the off-diagonal integral kernels of the fractional powers $A^{\alpha}$ in terms of the heat kernel expansion of $A$.

The study of the off-diagonal heat kernel $K\left(e^{-f(A) t} ; x, y\right)$ is motivated from two perspectives: first, it allows to approximately solve the evolution equation $\partial_{t} u(t)=f(A) u(t)$. Second, in probability theory one is interested in the integral $\mathbb{P}\left(X_{t} \in B\right)=\int_{B} K\left(e^{-t A} ; x, y\right) d y$ for measurable $B \subseteq M$. This gives the probability that a stochastic process $X$ with infinitesimal generator $A$ that starts at the point $x \in M$ at time 0 is in the set $B$ at time $t$. Also, it is the basis for Aronson-type estimates of the transition density.

The original motivation for this paper was to investigate the transition density for subordinate Brownian motion on $M$. Here $f$ is the Laplace exponent of the subordinator and $A$ is the infinitesimal generator of Brownian motion given by $\frac{1}{2} \Delta$. We illustrate this in Sect. 5 .

For symmetric processes on Euclidean space and Riemannian manifolds, there is a long tradition of upper and lower bounds of heat kernels leading to Aronson-type estimates, cf. $[12,17,18]$. There is also a strand of research that investigates jump processes on $\mathbb{R}^{n}$, see the survey [9] for comprehensive references and results. A more recent strand concerns jump processes living in bounded domains in Euclidean space with boundaries of different degrees of regularity. Exemplary investigations using sophisticated probabilistic techniques cover the special cases of fractional Laplacians $[8,10]$ and 
the relativistic stable processes [11]. The present paper can be regarded as a complement the latter investigations as we consider certain jump processes on closed manifolds.

Subordination in the sense of Bochner [7] allows to construct new stochastic processes from a given process using a random time change. On the level of generators of the corresponding semigroups, this translates to a functional calculus, cf. [24] for an early presentation and for example [27,28] for a general approach. We refer to $[22,29]$ for a comprehensive and historically exhaustive presentation.

The present paper considers subordinate Brownian motion using the calculus of classical pseudodifferential operators. This is also the viewpoint taken in $[14,15]$. These articles contain further references concerning the application of pseudodifferential operators in the context of certain stochastic processes. Noteworthy examples of this are the calculus developed in [20] and in the context of Weyl-Hörmander operators the paper [2] which allows variable-order subordination.

In the present paper, the geometry of the manifold is expressed by the coefficient functions in the heat kernel expansion of the Laplace-Beltrami operator. For completeness we mention that there is an alternative geometric viewpoint for stochastic processes on Euclidean space as suggested in [23]. This leads to estimates of the transition density of symmetric Lévy processes given in terms of a natural metric on the real line.

This paper is organised as follows. The following section collects some preliminaries from the theory of pseudodifferential operators. Sect. 3 motivates and states the key results, these are then proved in Sect. 4. Finally, Sect. 5 applies the approach to obtain estimates of the transition density for certain stochastic processes.

\section{Preliminaries and Notation}

For definitions of Sobolev spaces $H^{s}(M)$ and the calculus of (classical) pseudodifferential operators we refer the reader to [32]. We denote the space of pseudodifferential operators of order $r \in \mathbb{C}$ by $\Psi D O^{r}(M)$ and the subspace of classical operators by $\Psi D O_{c l}^{r}(M)$. In the notation of [32] this corresponds to $L_{1,0}^{r}(M)$ and $C L^{r}(M)$, respectively, with symbols from $S_{1,0}^{r}(M)$. For $Q \in$ $\Psi D O^{r}(M)$, we denote its Schwartz kernel by $K(Q ; x, y)$.

Define the smooth weight function $\langle x\rangle=\left(1+|x|^{2}\right)^{1 / 2}$ for $x \in \mathbb{R}^{n}$. The standard inner product in $L^{2}(M)$ is denoted by round brackets $(\cdot, \cdot)$. By $C^{l}(M)$ we denote the set of $l$-times continuously differentiable functions on $M$ and by $C^{\infty}(M)$ we denote the set of smooth functions on $M$. We define the partial differential operators $\partial_{j}=\frac{\partial}{\partial x_{j}}$ and for a multi-index $\beta=$ $\left(\beta_{1}, \ldots, \beta_{n}\right) \in \mathbb{N}_{0}^{n}$ we set $\partial_{x}^{\beta}=\partial_{1}^{\beta_{1}} \cdots \partial_{n}^{\beta_{n}}$.

Recall that one can form the complex powers and the heat operator as classical pseudodifferential operators. For the complex powers let $Q \in$ $\Psi D O_{c l}^{r}(M)$ with $r>0$ such that the resolvent set of $Q$ as an unbounded operator on $L^{2}(M)$ contains the half line $(-\infty, 0]$. Moreover assume 


$$
\left\|(Q-\lambda I)^{-1}\right\| \leq C(1+|\lambda|)^{-1}
$$

for any $\lambda \in(-\infty, 0]$ with operator norms on $L^{2}(M)$. Then it is a standard result that one can form a family of operators $Q^{z} \in \Psi D O_{c l}^{r z}(M)$, cf. [31,32].

For $Q \in \Psi D O_{c l}^{r}(M)$ with $r>0$ such that $Q$ is invertible and (2.1) holds in a sector $\{\lambda \in \mathbb{C} \mid \theta \leq \arg \lambda \leq 2 \pi-\theta\}$ for $\theta \in(\pi / 2, \pi)$, we can form the heat operator $e^{-t Q}$ for $t>0$ which belongs to $\Psi D O_{c l}^{-\infty}(M)$, cf. Section 5.6 in [1].

We recall the definition of asymptotic expansions of real-valued functions. Given a function $f:(0, \infty) \rightarrow \mathbb{R}$ we say that $f(t) \sim \sum_{k=0}^{\infty} a_{k} t^{\alpha_{k}}$ as $t \rightarrow 0^{+}$if

$$
\lim _{t \rightarrow 0^{+}} t^{-\alpha_{N}}\left(f(t)-\sum_{k=0}^{N} a_{k} t^{\alpha_{k}}\right)=0
$$

for some coefficients $a_{k} \in \mathbb{R}, \alpha_{k} \uparrow \infty$ and every $N \geq 0$. Similarly for $t \rightarrow \infty$. The analogous definition holds for Banach-space valued functions.

Also recall the $O$-notation: we say that $f(t)=O(g(t))$ as $t \rightarrow t_{0}$ if there is a constant $C$ such that $|f(t)| \leq C g(t)$ for any $t$ in a neighbourhood of $t_{0}$.

\section{Statement of the Key Results}

For ease of exposition and conscious of the probabilistic motivation we work with differential operators on closed manifolds, cf. Remark 3.8.

Assumptions. We summarise the key assumptions.

Hypothesis 3.1. Assume $A$ to be second-order differential operator such that

(i) $A$ is a generalised Laplacian: its principal symbol is given by the metric tensor;

(ii) $A$ is symmetric; and

(iii) the lowest eigenvalue of $A$ is strictly positive.

The final assumption is listed for convenience only: if $A$ has lowest eigenvalue 0 , then as in $\S 4$ of [31] we consider $A+\epsilon P$ instead where $\epsilon>0$ is small and $P$ is the projection onto the finite-dimensional kernel of $A$.

We recall the usual heat kernel asymptotics for generalised Laplacians.

Proposition 3.2. ([5], Theorem 2.30) Under Hypothesis 3.1 there are smooth functions $H_{0}, H_{1}, \ldots: M \times M \rightarrow \mathbb{R}$ such that

$$
K\left(e^{-t A} ; x, y\right) \sim\left(\frac{1}{2 \pi t}\right)^{n / 2} e^{-d(x, y)^{2} / 4 t} \sum_{k=0}^{\infty} H_{k}(x, y) t^{k}
$$

as $t \rightarrow 0^{+}$for $x, y \in M$. Here, $d(x, y)$ is the geodesic distance of $x, y \in M$.

Since we want to consider $f(A)$ defined by functional calculus we assume that the function $f$ has symbol-like properties.

Hypothesis 3.3. Let $f:[0, \infty) \rightarrow[0, \infty)$ be such that for some $\alpha \in(0,1)$ the following holds.

(i) The function $f$ is smooth;

(ii) For any $l \in \mathbb{N}_{0}$ there is a constant $C_{l}$ with $\left|\partial_{\lambda}^{l} f(\lambda)\right| \leq C_{l}\langle\lambda\rangle^{\alpha-l}$ for all $\lambda \geq 0$ 
(iii) The map $f$ has the asymptotic expansion

$$
f(\lambda) \sim m+a_{0} \lambda^{\alpha}+a_{1} \lambda^{\alpha-1}+a_{2} \lambda^{\alpha-2}+\cdots
$$

as $\lambda \rightarrow \infty$ with $a_{0}, a_{1}, \ldots \in \mathbb{R}$ and $m<0$, i.e. strictly negative.

Example. The key example we have in mind is $f(\lambda)=(1+\lambda)^{\alpha}-1$, which is probabilistically significant: the operator $f\left(\frac{1}{2} \Delta\right)$ is the infinitesimal generator of the relativistic $\alpha$-stable process, cf. Sect. 5 . Here

$$
f(\lambda) \sim-1+\lambda^{\alpha}+\alpha \lambda^{\alpha-1}+\frac{\alpha(\alpha-1)}{2} \lambda^{\alpha-2}+\cdots
$$

as $\lambda \rightarrow \infty$ by Taylor's theorem. More probabilistically motivated functions satisfying Hypothesis 3.3 can be found in Example 1 of [14].

Kernels of the complex powers. Our first key result gives a representation of the kernels $K\left(A^{-z} ; x, y\right)$ as an asymptotic series in $d(x, y)$ for small distances.

Theorem 3.4. Let $A$ be an operator satisfying Hypothesis 3.1 and choose $x, y \in$ $M$ with $x \neq y$. Then there is a function $F(w ; x, y): \mathbb{C} \times M \times M \rightarrow \mathbb{C}$ such that for any $z \in \mathbb{C}$ the following asymptotics of the kernels of the complex powers $A^{-z}$ hold as $d(x, y) \rightarrow 0$.

(i) If $z-n / 2 \notin \mathbb{Z}$, then

$$
\begin{aligned}
\Gamma(z) K\left(A^{-z} ; x, y\right) \sim & \sum_{k=0}^{\infty} \frac{H_{k}(x, y)}{(2 \pi)^{n / 2}} \frac{\Gamma\left(\frac{n}{2}-k-z\right)}{\left(\frac{1}{4} d(x, y)^{2}\right)^{\frac{n}{2}-k-z}} \\
& +\sum_{k=0}^{\infty} F(z-k ; x, y) \frac{(-1)^{k}}{k !}\left(\frac{1}{4} d(x, y)^{2}\right)^{k}
\end{aligned}
$$

(ii) If $z=n / 2-N$ for some $N \in \mathbb{N}_{0}$, then

$$
\begin{aligned}
\Gamma(z) K\left(A^{-z} ; x, y\right) \sim & \sum_{k=0}^{N-1} \frac{H_{k}(x, y)}{(2 \pi)^{n / 2}} \frac{\Gamma(N-k)}{\left(\frac{1}{4} d(x, y)^{2}\right)^{N-k}} \\
& -\sum_{k=N}^{\infty} \frac{H_{k}(x, y)}{(2 \pi)^{n / 2}} \frac{(-1)^{N-k}}{(N-k) !}\left(\frac{1}{4} d(x, y)^{2}\right)^{N-k} \log \frac{1}{4} d(x, y)^{2} ;
\end{aligned}
$$

(iii) If $z=n / 2+N$ for some $N \in \mathbb{N}_{0}$, then

$$
\begin{aligned}
\Gamma(z) K\left(A^{-z} ; x, y\right) \sim & \sum_{k=0}^{N-1} F(z-k ; x, y) \frac{(-1)^{k}}{k !}\left(\frac{1}{4} d(x, y)^{2}\right)^{k} \\
& -\sum_{k=N}^{\infty} \frac{H_{k}(x, y)}{(2 \pi)^{n / 2}} \frac{(-1)^{k}}{k !}\left(\frac{1}{4} d(x, y)^{2}\right)^{k} \log \frac{1}{4} d(x, y)^{2} .
\end{aligned}
$$

In each case the functions $H_{k}$ are from (3.1).

The relation $\sim$ denotes an asymptotic series in powers of $d(x, y)$, and we refer to (4.8) for explicit bounds of remainder terms.

For completeness, we compare this to the kernel expansion of pseudodifferential operators of negative order on $\mathbb{R}^{n}$. Proposition 2.8 of [34], which 
we quote almost verbatim, allows to write the kernel of $Q \in \Psi D O_{c l}^{-j}\left(\mathbb{R}^{n}\right)$ for $j \in \mathbb{N}$ asymptotically in any $C^{l}\left(\mathbb{R}^{n} \times \mathbb{R}^{n}\right)$ as

$$
K(Q ; x, y) \sim \sum_{k=0}^{\infty} q_{k}(x, x-y)+p_{k}(x, x-y) \log |x-y| .
$$

Here, the $q_{k}$ are such that any $D_{x}^{\beta} q_{k}$ is a bounded continuous function of $x$ with values in homogeneous distributions of degree $j+k-n$ that are smooth on $\mathbb{R}^{n} \backslash\{0\}$. The $p_{k}$ are polynomials homogeneous of degree $j+k-n$ in $x-y$ with coefficients that are bounded continuous functions of $x$ together with all their $x$-derivatives.

Heat kernel asymptotics. Our second key result gives the asymptotics of the heat kernels of $f(A)$.

Theorem 3.5. Assume that the closed manifold $M$ has dimension $n>4-2 \alpha$. Let $A$ be a differential operator satisfying Hypotheses 3.1 and suppose that $f$ is a function satisfying Hypothesis 3.3. Then for $x, y \in M$ with $x \neq y$ the following holds.

(i) Let $K_{1}$ and $K_{2}$ be compact and disjoint subsets of $M$. Under Hypotheses 3.1 and 3.3 we have the heat kernel asymptotics

$$
K\left(e^{-f(A) t} ; x, y\right) \sim e^{-m t} \sum_{k=1}^{\infty} \frac{(-1)^{k}}{k !} K\left((f(A)-m I)^{k} ; x, y\right) t^{k}
$$

as $t \rightarrow 0^{+}$in $C\left(K_{1} \times K_{2}\right)$, where $m$ is from (3.2).

(ii) In the lowest order in $t$ we have

$$
K\left(e^{-t f(A)} ; x, y\right) \sim t e^{-m t}\left[H(x, y)+O\left(d(x, y)^{-n-2 \alpha+4}\right)\right]
$$

as $t \rightarrow 0^{+}$where

$$
\begin{aligned}
H(x, y)= & \frac{\Gamma\left(\frac{n}{2}+\alpha\right) 4^{n / 2+\alpha}}{\Gamma(1-\alpha)(2 \pi)^{n / 2}} \cdot \frac{\alpha a_{0} H_{0}(x, y)}{d(x, y)^{n+2 \alpha}} \\
& +\frac{\Gamma\left(\frac{n}{2}+\alpha-1\right) 4^{n / 2+\alpha-2}}{\Gamma(1-\alpha)(2 \pi)^{n / 2}} \cdot \frac{\alpha a_{0} H_{1}(x, y)-a_{1} H_{0}(x, y)}{d(x, y)^{n+2 \alpha-2}}
\end{aligned}
$$

and $H_{0}, H_{1}$ are from the heat kernel expansion (3.1).

Remark 3.6. Note that the expansion (3.3) is rather suggestive. The right hand side is the power series for the exponential function of $f(A)-m I$ with the lowest-order term removed. Formally in terms of operators

$$
e^{-f(A) t}=e^{-m t} e^{-(f(A)-m) t}=e^{-m t} \sum_{k=0}^{\infty} \frac{(-1)^{k}}{k !}(f(A)-m I)^{k} t^{k},
$$

so that (3.3) rigorously phrases this in terms of integral kernels.

An immediate corollary gives an upper bound for the heat kernel.

Corollary 3.7. Under the assumptions of Theorem 3.5 we have the bound

$$
K\left(e^{-f(A) t} ; x, y\right) \leq C t e^{-m t}\left(\left(d(x, y)+t^{1 / 2 \alpha}\right)^{-n-2 \alpha} \wedge H(x, y)\right)
$$


for $t \in(0,1)$ and $a$ constant $C>0$. Here, $a \wedge b$ denotes the minimum of $a$ and $b$.

Remark 3.8. The setup and results can be easily generalised to pseudodifferential operators and more general manifolds. One could apply the same approach to a hypoelliptic operator $A$ on a Heisenberg manifold [25] with heat kernel expansion as in [4]. The heat kernel of $f(A)$ would have the probabilistic interpretation of the transition density for a subordinate process.

\section{Proof of the Key Results}

The main idea in deriving the heat kernel asymptotics of $f(A)$ is to exploit the well-known correspondence between the heat kernel asymptotics and the pole structure of the kernel of the complex powers $f(A)^{-z}$. This correspondence is implemented by the Mellin transform.

\subsection{Relation Between the Heat Kernel and Complex Powers}

First recall the Mellin transform which turns a function $f:[0, \infty) \rightarrow \mathbb{C}$ into

$$
\mathcal{M}[f ; z]=\int_{0}^{\infty} f(t) t^{z-1} d t
$$

for $z \in \mathbb{C}$ whenever the integral makes sense. The Mellin transform is analytic in the strip $\alpha<\operatorname{Re} z<\beta$ where

$$
\begin{aligned}
& \alpha=\inf \left\{\alpha^{*} \mid f(t)=O\left(t^{-\alpha^{*}}\right) \text { as } t \rightarrow 0^{+}\right\} \\
& \beta=\sup \left\{\beta^{*} \mid f(t)=O\left(t^{-\beta^{*}}\right) \text { as } t \rightarrow \infty\right\} .
\end{aligned}
$$

Often the Mellin transform can be extended meromorphically beyond this strip of analyticity (there is an analogous statement for $t \rightarrow \infty$ ).

Lemma 4.1. Suppose the function $f:(0, \infty) \rightarrow \mathbb{C}$ has the asymptotics

$$
f(t) \sim \sum_{m=0}^{\infty} \sum_{k=0}^{N(m)} p_{m k}(\log t)^{k} t^{a_{m}}
$$

as $t \rightarrow 0^{+}$with $\operatorname{Re} a_{m} \uparrow \infty$ and $N(m) \geq 0$ finite for each $m$. Then $\alpha=$ $-\operatorname{Re} a_{0}$ and $\mathcal{M}[f ; z]$ can be analytically continued as a meromorphic function into the left half plane Re $z \leq \alpha$ with poles at the points $z=-a_{m}$. This extension has a Laurent expansion about $z=-a_{m}$ with singular part

$$
\sum_{k=0}^{N(m)} \frac{p_{m k}(-1)^{k} k !}{\left(z+a_{m}\right)^{k+1}} .
$$

Moreover, for any $x<\beta$ we have $\lim _{|y| \rightarrow \infty} \mathcal{M}[f ; x+i y]=0$.

The converse also holds, so that (4.1) and (4.2) are equivalent.

Proof. This is standard: cf. Lemma 4.3 .6 of [6] for the implication (4.1) $\Rightarrow$ (4.2) and $\S 3.3 .3 .2$ of [30] for the converse. 
The correspondence between heat kernel asymptotics and properties of the complex powers of an operator is based on the relation

$$
\Gamma(z) K\left(Q^{-z} ; x, y\right)=\mathcal{M}\left[K\left(e^{-t Q} ; x, y\right) ; z\right],
$$

for $\operatorname{Re} z$ suitably large where $\Gamma$ is the Gamma function. This relation is typically exploited to investigate the on-diagonal behaviour of the heat kernel, see for example Section 5.5 of [1]. Lemma 4.1 allows us to link the singular structure of the kernel of the complex powers and the heat kernel asymptotics.

Corollary 4.2. Let $Q \in \Psi D O_{c l}^{r}(M)$ be self-adjoint and elliptic such that the complex powers $Q^{-z}$ exist in $\Psi D O_{c l}^{-r z}(M)$. Fix $x, y \in M$ and let $z \in \mathbb{C}$ with Re $z>n / r$. If $\Gamma(z) K\left(Q^{-z} ; x, y\right)$ has the singularity structure

$$
\sum_{k=0}^{\infty} \frac{c_{k}(x, y)}{z-l_{k}}+\sum_{k=1}^{\infty} \frac{c_{k}^{\prime}(x, y)}{(z-k)^{2}}
$$

for functions $c_{k}, c_{k}^{\prime}: M \times M \rightarrow \mathbb{C}$ and complex numbers $l_{k}$, then the heat kernel $K\left(e^{-t Q} ; x, y\right)$ has the asymptotic expansion

$$
K\left(e^{-t Q} ; x, y\right) \sim \sum_{k=0}^{\infty} c_{k}(x, y) t^{-l_{k}}+\sum_{k=1}^{\infty} c_{k}^{\prime}(x, y) t^{k} \log t
$$

as $t \rightarrow 0$. Double poles of $\Gamma(z) K\left(Q^{-z} ; x, y\right)$ lead to logarithmic terms in the heat kernel expansion.

Proof. Let $\mu_{k}$ be the eigenvalues of $Q$ in increasing order with corresponding orthonormal basis $\varphi_{k}$ of $L^{2}(M)$ consisting of eigenfunctions. We then have

$$
K\left(e^{-t Q} ; x, y\right)=\sum_{k=1}^{\infty} e^{-\mu_{k} t} \varphi_{k}(x) \varphi_{k}(y)
$$

and

$$
K\left(Q^{-z} ; x, y\right)=\sum_{k=1}^{\infty} \mu_{k}^{-z} \varphi_{k}(x) \varphi_{k}(y) .
$$

So $\Gamma(z) K\left(Q^{-z} ; x, y\right)=\int_{0}^{\infty} K\left(e^{-t Q} ; x, y\right) t^{z-1} d t$ and the claim follows from Lemma 4.1.

\subsection{Asymptotics of the Kernels of the Complex Powers}

We first define two transforms

$$
\begin{aligned}
& F(w ; x, y)=\mathcal{M}\left[K\left(e^{-A t} ; x, y\right) e^{d(x, y)^{2} / 4 t} ; w\right], \\
& G(w ; x, y)=\mathcal{M}\left[e^{-d(x, y)^{2} / 4 t} ; w\right],
\end{aligned}
$$

where we omit the $x, y$-dependence in the following to ease the presentation.

Each of these functions is meromorphic in $w$ with explicit pole structure.

Lemma 4.3. Fix $x, y \in M$ with $x \neq y$. Then the following holds. 
(i) The map $F(w)$ is analytic for Re $w>n / 2$. It can be meromorphically extended to the complex plane with at most simple poles in the set

$$
\mathcal{P}_{F}=\left\{\frac{n}{2}, \frac{n}{2}-1, \frac{n}{2}-2, \ldots\right\} .
$$

The residue of $F$ at $\frac{n}{2}-k$ is given by $\frac{H_{k}(x, y)}{(2 \pi)^{n / 2}}$.

(i) For given $z \in \mathbb{C}$ the map $G(z-w)$ can be evaluated as

$$
G(z-w)=\left(\frac{1}{4} d(x, y)^{2}\right)^{z-w} \Gamma(-(z-w)) .
$$

It is analytic for $\operatorname{Re} w>\operatorname{Re} z$ and can be meromorphically continued to the complex plane with simple poles in the set

$$
\mathcal{P}_{G}=\{z, z-1, z-2, \ldots\} .
$$

The residue of $G(z-w)$ at $w=z-k$ is given by $\frac{(-1)^{k}}{k !}\left(\frac{1}{4} d(x, y)^{2}\right)^{k}$.

In summary, the map $w \mapsto F(w) G(z-w)$ is analytic the half plane Re $w>$ $\max \left\{\frac{n}{2}, \operatorname{Re} z\right\}$ and can be extended to a meromorphic function with poles at points in the discrete set $\mathcal{P}_{F} \cup \mathcal{P}_{G}$. The poles at points in the intersection $\mathcal{P}_{F} \cap \mathcal{P}_{G}$ are double poles, all other poles are at most simple.

Proof. First consider $F(w)$ : the kernel $K\left(e^{-A t} ; x, y\right) e^{d(x, y)^{2} / 4 t}$ can asymptotically be expressed in powers of $t$ for $t \rightarrow 0^{+}$as

$$
K\left(e^{-A t} ; x, y\right) e^{d(x, y)^{2} / 4 t} \sim \frac{H_{0}(x, y)}{(2 \pi)^{n / 2}} t^{-n / 2}+\frac{H_{1}(x, y)}{(2 \pi)^{n / 2}} t^{-n / 2+1}+\cdots,
$$

so that the strip of analyticity is bounded to the left by $n / 2<\operatorname{Re} w$. Note that $K\left(e^{-A t} ; x, y\right) e^{d(x, y)^{2} / 4 t}$ decays like $e^{-\mu_{1} t}$ as $t \rightarrow \infty$ with $\mu_{1}>0$ the lowest eigenvalue of $A$. So this function decays faster than any polynomial, and hence the Mellin transform is analytic on the strip $n / 2<\operatorname{Re} w<\infty$.

By Lemma 4.1, the Mellin transform $F$ of $K\left(e^{-t A} ; x, y\right)$ extends meromorphically to the left-half plane $\operatorname{Re} w<n / 2$ with simple poles in the set

$$
\mathcal{P}_{F}=\left\{\frac{n}{2}, \frac{n}{2}-1, \frac{n}{2}-2, \ldots\right\} .
$$

The corresponding residues are given as $\frac{H_{0}(x, y)}{(2 \pi)^{n / 2}}, \frac{H_{1}(x, y)}{(2 \pi)^{n / 2}}, \ldots$ which follows from (4.5).

Second, for fixed $z \in \mathbb{C}$ the transform $\mathcal{M}\left[e^{-d(x, y)^{2} / 4 t} ; z-w\right]$ can be explicitly computed as

$$
\mathcal{M}\left[e^{-d(x, y)^{2} / 2 t} ; z-w\right]=\left(\frac{1}{4} d(x, y)^{2}\right)^{z-w} \Gamma(-(z-w))
$$

by the usual integral representation of the Gamma function and a change of variables. It is meromorphic on $\mathbb{C}$ with simple poles at the points in the set

$$
\mathcal{P}_{G}=\{z, z-1, z-2, \ldots\},
$$

the residues are given by standard properties of the Gamma function.

We also bound certain integrals that later appear as error terms. 
Lemma 4.4. For $z \in \mathbb{C}$ with $\operatorname{Re} z \neq c$ there is a constant $C$ such that

$$
\left|\frac{1}{2 \pi i} \int_{c-i \infty}^{c+i \infty} F(w) G(z-w) d w\right| \leq C\left(\frac{1}{4} d(x, y)^{2}\right)^{-(c-\operatorname{Re} z)} .
$$

The constant $C$ depends on $z$ and $c$.

Proof. Recall the upper bound of the Gamma function

$$
|\Gamma(x+i y)| \leq C^{\prime}|y|^{x-1 / 2} e^{-\pi|y| / 2}
$$

for some constant $C^{\prime}$, cf. Chapter 2.4.3 of [26]. We thus find

$$
\begin{aligned}
& \left|\frac{1}{2 \pi i} \int_{c-i \infty}^{c+i \infty} F(w) G(z-w) d w\right| \\
\leq & \frac{1}{2 \pi} \int_{c-i \infty}^{c+i \infty}|F(w)|\left|\frac{\Gamma(w-z)}{\left(\frac{1}{4} d(x, y)^{2}\right)^{w-z}}\right| d w \\
\leq & \frac{1}{2 \pi} \int_{c-i \infty}^{c+i \infty}|F(w)| \frac{C^{\prime}|\operatorname{Im}(w-z)|^{c-\operatorname{Re} z-1 / 2} e^{-\pi|\operatorname{Im}(w-z)| / 2}}{\left(\frac{1}{4} d(x, y)^{2}\right)^{c-\operatorname{Re} z}} d w .
\end{aligned}
$$

The Mellin transform $F(w)$ goes to zero as $|\operatorname{Im} w| \rightarrow \infty$ by Lemma 4.1 so that the integral can be bounded in norm. By the definition of $F$ this bound may depend on $x, y$ through the functions $d$ and $H_{0}, H_{1}, \ldots$ However, these are bounded as $M$ is compact and the maps are smooth. Overall, we can bound the integral in terms of a constant depending on $c$ and $z$.

We are now ready to prove the theorem concerning the kernels of the complex powers.

Proof of Theorem 3.4. Starting with the formal relation

$$
\Gamma(z) K\left(A^{-z} ; x, y\right)=\int_{0}^{\infty} K\left(e^{-t A} ; x, y\right) t^{z-1} d t
$$

we first establish that this is valid for any $z \in \mathbb{C}$ and then approximate the right hand side in powers of $d(x, y)$.

1. Observe that the right hand side of (4.7) defines an entire function in $z$ : as $t \rightarrow 0^{+}$, the kernel $K\left(e^{-t A} ; x, y\right)$ is of rapid decay due to the factor of $e^{-d(x, y)^{2} / 4 t}$ in (3.1) and as $t \rightarrow \infty$, the kernel is of rapid decay as the lowest eigenvalue of $A$ is positive.

The kernel $K\left(A^{-z} ; x, y\right)$ in the sense of the series representation (4.4) makes sense for $\operatorname{Re} z>n / 2$ and can be analytically continued to an entire function in $z$, cf. Theorem 12.1 of [32]. The zeros of $K\left(A^{-z} ; x, y\right)$ cancel the poles of the Gamma function.

Overall, the relation (4.7) is valid for any $z \in \mathbb{C}$.

2. We now expand the right hand side of (4.7) in powers of $d(x, y)$. First recall the Parseval formula for the Mellin transform ([35], Chapter II.2.1.):

$$
\int_{0}^{\infty} f(t) g(t) d t=\frac{1}{2 \pi i} \int_{c-i \infty}^{c+i \infty} \mathcal{M}[f(t) ; w] \mathcal{M}[g(t) ; 1-w] d w
$$


where the vertical line $c+i \mathbb{R}$ lies in the intersection of the strips of analyticity of the Mellin transforms of $f$ and $g$. We formally apply this to obtain

$$
\begin{aligned}
\Gamma(z) & K\left(A^{-z} ; x, y\right) \\
= & \int_{0}^{\infty} K\left(e^{-A t} ; x, y\right) e^{d(x, y)^{2} / 4 t} \cdot e^{-d(x, y)^{2} / 4 t} t^{z-1} d t \\
= & \frac{1}{2 \pi i} \int_{c-i \infty}^{c+i \infty} \mathcal{M}\left[K\left(e^{-A t} ; x, y\right) e^{d(x, y)^{2} / 4 t} ; w\right] \mathcal{M}\left[e^{-d(x, y)^{2} / 4 t} t^{z-1} ; 1-w\right] d w \\
= & \frac{1}{2 \pi i} \int_{c-i \infty}^{c+i \infty} \mathcal{M}\left[K\left(e^{-A t} ; x, y\right) e^{d(x, y)^{2} / 4 t} ; w\right] \mathcal{M}\left[e^{-d(x, y)^{2} / 4 t} ; z-w\right] d w \\
= & \frac{1}{2 \pi i} \int_{c-i \infty}^{c+i \infty} F(w) G(z-w) d w
\end{aligned}
$$

with $c$ to be determined: by Lemma 4.3, the integrand is analytic on the half-plane $\operatorname{Re} w>\max \left\{\frac{n}{2}, \operatorname{Re} z\right\}$ so we choose $c$ such that the contour $c+i \mathbb{R}$ lies in this half-plane.

3. The idea now is to repeatedly apply Cauchy's residue theorem and to move the contour across the poles of the integrand. Shifting the contour is allowed since the integrand $F(w) G(z-w)$ goes to zero as $|\operatorname{Im} w| \rightarrow \infty$ for fixed $z$. This is for two reasons: the Mellin transform $F(w)$ goes to zero as $|\operatorname{Im} w| \rightarrow \infty$ by Lemma 4.1 and we have (4.6) bounding $G$.

Case I: only simple poles. Here we assume that $z-n / 2 \notin \mathbb{Z}$ so that the sets of poles of $F$ and $G$ do not overlap. We move the contour $c+i \mathbb{R}$ to the left to a contour $c^{\prime}+i \mathbb{R}$ with $c^{\prime}<c$ thereby possibly crossing poles. The contour $c^{\prime}+i \mathbb{R}$ itself must avoid any poles. This leads to

$$
\begin{aligned}
& \frac{1}{2 \pi i} \int_{c-i \infty}^{c+i \infty} F(w) G(z-w) d w \\
& =\underbrace{\sum_{k: \frac{n}{2}-k>c^{\prime}} \frac{\Phi_{k}(x, y)}{\left(\frac{1}{4} d(x, y)^{2}\right)^{n / 2-k-z}}+\underbrace{\sum_{k: z-k>c^{\prime}} \Psi_{k}(x, y)\left(\frac{1}{4} d(x, y)^{2}\right)^{k}}_{\text {poles of } G(w-z) \text { to the right of } c^{\prime}+i \mathbb{R}}+R(x, y)}_{\text {poles of } F(w) \text { to the right of } c^{\prime}+i \mathbb{R}}
\end{aligned}
$$

where

$$
\begin{array}{r}
\Phi_{k}(x, y)=\Gamma\left(\frac{n}{2}-k-z\right), \quad \Psi_{k}(x, y)=F(z-k) \frac{(-1)^{k}}{k !}, \\
R(x, y)=\frac{1}{2 \pi i} \int_{c^{\prime}-i \infty}^{c^{\prime}+i \infty} F(w) G(z-w) d w .
\end{array}
$$

By Lemma 4.4, the error term $R$ can be bounded as

$$
|R(x, y)| \leq C\left(\frac{1}{4} d(x, y)^{2}\right)^{-\left(c^{\prime}-\operatorname{Re} z\right)}
$$

for a constant $C$. This can be recast in more familiar terms of asymptotic expansions: given $c^{\prime} \in \mathbb{R}$ such that the contour $c^{\prime}+i \mathbb{R}$ avoids any poles in $\mathcal{P}_{F} \cup \mathcal{P}_{g}$, there are natural numbers $N_{1}, N_{2}$ such that 


$$
\begin{aligned}
& \left|\Gamma(z) K\left(A^{-z} ; x, y\right)-\sum_{k=0}^{N_{1}} \frac{\Phi_{k}(x, y)}{\left(\frac{1}{4} d(x, y)^{2}\right)^{n / 2-k-z}}+\sum_{k=0}^{N_{2}} \Psi_{k}(x, y)\left(\frac{1}{4} d(x, y)^{2}\right)^{k}\right| \\
& \quad \leq C\left(\frac{1}{4} d(x, y)^{2}\right)^{\operatorname{Re} z-c^{\prime}} .
\end{aligned}
$$

More precisely, we set $N_{1}=\sup \left\{k \mid \frac{n}{2}-k>c^{\prime}\right\}$ and $N_{2}=\sup \left\{k \mid \operatorname{Re} z-k>c^{\prime}\right\}$. If the set over which the supremum is taken is empty, i.e. if there are no poles of $F$ or $G$ to the right of the contour $c^{\prime}+i \mathbb{R}$, then we understand the corresponding sums to be zero.

Case II: double poles. First, we assume that $z=n / 2-N$ for some $N \in \mathbb{N}_{0}$. This means that the rightmost pole of $F(w) G(z-w)$ is located at $n / 2$. Thus, if we start with a contour $c+i \mathbb{R}$ to the right of $n / 2$ and move this to the left we will cross the pole(s) of $F$ first before encountering the double poles. If $z=n / 2-N$, then we will have only finitely many simple poles: they are due to $F$ and are located at points of the set $\left\{\frac{n}{2}, \frac{n}{2}-1, \ldots, \frac{n}{2}-(N-1)\right\}$. All other poles are double poles and are located at points in the set $\left\{\frac{n}{2}-\right.$ $\left.N, \frac{n}{2}-(N+1), \ldots\right\}$.

Suppose that we have crossed all simple poles of $F$ so that we integrate along a contour $c^{\prime}+i \mathbb{R}$ with $c^{\prime}<n / 2-N$. We pick up all simple poles of $F$ plus the double poles at $n / 2-k$ which lie to the right of $c^{\prime}+i \mathbb{R}$, i.e. for which $n / 2-k>c^{\prime}$. We thus obtain

$$
\begin{aligned}
\frac{1}{2 \pi i} \int_{c-i \infty}^{c+i \infty} F(w) G(z-w) d w \\
=\underbrace{\sum_{k=0}^{N-1} \frac{\Phi_{k}(x, y)}{\left(\frac{1}{4} d(x, y)^{2}\right)^{n / 2-k-z}}}_{\text {simple poles to the right of } c^{\prime}+i \mathbb{R}} \\
+\underbrace{\sum_{N \leq k<\frac{n}{2}-c^{\prime}} \text { res }_{w=\frac{n}{2}-k} F(w) G(z-w)}_{\text {double poles to the right of } c^{\prime}+i \mathbb{R}}+R(x, y)
\end{aligned}
$$

with $\Phi_{k}$ and $R$ as above. To compute the residue at the double poles note that in a neighbourhood of $w_{k}=n / 2-k$ we have

$$
F(w) G(z-w)=\frac{H_{k}(x, y)}{(2 \pi)^{n / 2}} \frac{(-1)^{N-k}}{(N-k) !} \frac{g(w)}{\left(w-w_{k}\right)^{2}}+\text { holo }
$$

where holo denotes a holomorphic function which is of no concern here and

$$
g(w)=\left(\frac{1}{4} d(x, y)^{2}\right)^{-w+n / 2-N} .
$$

Cauchy's theorem for derivatives gives

$$
\begin{aligned}
& \underset{\substack{\operatorname{res} \\
w=\frac{n}{2}-k}}{ } F(w) G(z-w) \\
& =\frac{H_{k}(x, y)}{(2 \pi)^{n / 2}} \frac{(-1)^{N-k}}{(N-k) !} g^{\prime}\left(w_{k}\right)
\end{aligned}
$$




$$
=\frac{H_{k}(x, y)}{(2 \pi)^{n / 2}} \frac{(-1)^{N-k}}{(N-k) !} \cdot\left[-\left(\frac{1}{4} d(x, y)^{2}\right)^{k-N} \cdot \log \frac{1}{4} d(x, y)^{2}\right],
$$

which proves the claim.

It remains to consider the alternative $z=n / 2+N$ for some $N \in \mathbb{N}_{0}$, whereupon the rightmost poles of $F(w) G(z-w)$ are simple poles of $G(z-w)$. The argument is almost verbatim as before.

\subsection{Heat Kernel Asymptotics}

To analyse the heat kernel asymptotics of $f(A)$ we expand $f(A)$ in fractional powers of $A$ to write $f(A)=a_{0} A^{\alpha}+a_{1} A^{\alpha-1}+\cdots$ and then use the kernel asymptotics of these powers.

Proposition 4.5. Under the above hypotheses, the following assertions hold.

(i) The operator $f(A)-m I$ belongs to $\Psi D O_{c l}^{2 \alpha}(M)$.

(ii) There is a pseudodifferential operator $R_{2 \alpha-4} \in \Psi D O_{c l}^{2 \alpha-4}(M)$ such that

$$
f(A)=m I+a_{0} A^{\alpha}+a_{1} A^{\alpha-1}+R_{2 \alpha-4} .
$$

(iii) The complex powers $(f(A)-m I)^{-z}$ belong to $\Psi D O_{c l}^{-2 \alpha z}(M)$.

(iv) For $x, y \in M$ with $x \neq y$, the kernel $K\left((f(A)-m I)^{-z} ; x, y\right)$ of the complex powers can be analytically continued to an entire function in $z$.

Remark 4.6. If $Q=f(A)-m I$ were a differential operator, then the $\mathrm{k}$ ernel $K\left(Q^{-z} ; x, y\right)$ would vanish at $z=0,-1,-2, \ldots$ since then the operator $Q^{-(-l)}=Q^{l}$ is a differential operator and hence local. To see this, let $u, v \in C^{\infty}(M)$ with disjoint support. Then in $L^{2}(M)$ the inner product $\left(Q^{l} u, v\right)=0$. Since this holds for any such $u, v$, the kernel of $Q^{l}$ must be zero off-diagonal, cf. step 4 of the proof of Theorem 12.1 in [32]. In general, $f(A)-m I$ is not local so that we expect $K\left((f(A)-m I)^{-z} ; x, y\right)$ to be nonzero at $z$ from the nonpositive integers.

Proof. (i) The fact that $f(A)-m I \in \Psi D O^{2 \alpha}(M)$ follows from functional calculus, cf. [13], Theorem 1 of [33] or Theorem 1 of [19].

To show that the operator is classical, define an operator $B_{N}$ as a finite sum of classical pseudodifferential operators

$$
B_{N}=a_{0} A^{\alpha}+a_{1} A^{\alpha-1}+a_{2} A^{\alpha-2}+\cdots+a_{N} A^{\alpha-N} .
$$

Let $\left\{\varphi_{k}\right\}$ be an orthonormal basis of $L^{2}(M)$ consisting eigenfunctions of $A$ with corresponding eigenvalues $\left\{\mu_{k}\right\}$. We have

$$
(f(A)-m I) \varphi_{k}=\left(f\left(\mu_{k}\right)-m \mu_{k}\right) \varphi_{k}
$$

and

$$
B_{N} \varphi_{k}=\left(a_{0} \mu_{k}^{\alpha}+\cdots+a_{N} \mu_{k}^{\alpha-N}\right) \varphi_{k} .
$$

So we find in $L^{2}(M)$ that

$$
\begin{aligned}
& \left\|\left[(f(A)-m I)-B_{N}\right] \varphi_{k}\right\| \\
& \quad=\left\|\left[\left(f\left(\mu_{k}\right)-m \mu_{k}\right)-\left(a_{0} \mu_{k}^{\alpha}+\cdots+a_{N} \mu_{k}^{\alpha-N}\right)\right] \varphi_{k}\right\|
\end{aligned}
$$




$$
\begin{aligned}
& =\left|\left(f\left(\mu_{k}\right)-m \mu_{k}\right)-\left(a_{0} \mu_{k}^{\alpha}+\cdots+a_{N} \mu_{k}^{\alpha-N}\right)\right| \\
& \leq C_{N}\left|\mu_{k}\right|^{\alpha-N-1},
\end{aligned}
$$

for some constant $C_{N}$ independent of $k$ where the last inequality follows from (3.2). Thus, $A^{-\alpha+N+1}\left[(f(A)-m I)-B_{N}\right] \in \Psi D O^{0}(M)$ or $(f(A)-m I)-$ $B_{N} \in \Psi D O^{2 \alpha-2(N+1)}(M)$. Letting $N \rightarrow \infty$ in the equation for $B_{N}$ and asymptotically summing (Proposition 3.5 in Chapter I of [32]) we obtain an operator

$$
B=a_{0} A^{\alpha}+a_{1} A^{\alpha-1}+a_{2} A^{\alpha-2}+\cdots
$$

which belongs to $\Psi D O_{c l}^{2 \alpha}$. Moreover, $(f(A)-m I)-B \in \Psi D O^{-\infty}(M)$ so that $f(A)-m I \in \Psi D O_{c l}^{2 \alpha}(M)$ and is classical.

(ii) This follows from the construction of $f(A)-m I$ itself.

(iii) and (iv) follow from Theorem 5.5.1 of [1].

Remark 4.7. For technical reasons we consider $f(A)-m I$ instead of $f(A)$ since the former is a classical pseudodifferential operator whereas the latter is not classical: the symbol can be expanded into homogeneous terms with orders $2 \alpha-k$ for $k=0,1,2, \ldots$ plus a term of order 0 (a multiple of the identity), so the difference in orders is not an integer.

We proceed to the heat kernel asymptotics of $f(A)-m I$, viz. assertion (i) of Theorem 3.5.

Proposition 4.8. Let $K_{1}$ and $K_{2}$ be compact and disjoint subsets of $M$. Under Hypotheses 3.1 and 3.3 we have

$$
K\left(e^{-f(A) t} ; x, y\right) \sim e^{-m t} \sum_{k=1}^{\infty} \frac{(-1)^{k}}{k !} K\left((f(A)-m I)^{k} ; x, y\right) t^{k}
$$

as $t \rightarrow 0^{+}$in $C\left(K_{1} \times K_{2}\right)$.

Proof. We start with the equation

$$
\Gamma(z) K\left((f(A)-m I)^{-z} ; x, y\right)=\int_{0}^{\infty} K\left(e^{-(f(A)-m I) t} ; x, y\right) t^{z-1} d t .
$$

The integral on the right hand side is absolutely convergent for $\operatorname{Re} z>-1$ : the kernel $K\left(e^{-(f(A)-m I) t} ; x, y\right)$ decreases exponentially for $t \rightarrow \infty$ due to the lowest eigenvalue being positive and it is bounded as $t \rightarrow 0$ of order $t$ by Theorem 1 of [16], equation $(*)$. The left hand side can be extended to a meromorphic function in the entire complex plane with at most simple poles at the points $z=0,-1,-2, \ldots$ that correspond to the poles of the Gamma function as $K\left((f(A)-m I)^{-z} ; x, y\right)$ is entire. This yields the unique meromorphic continuation of the right hand side.

For $\operatorname{Re} z>n / 2 \alpha$ we invert the Mellin transform to write

$$
K\left(e^{-(f(A)-m I) t} ; x, y\right)=\frac{1}{2 \pi i} \int_{c-i \infty}^{c+i \infty} \Gamma(z) K\left((f(A)-m I)^{-z} ; x, y\right) t^{-z} d z
$$

and repeat the method of the proof of Theorem 3.4. Here, $c+i \mathbb{R}$ is a contour chosen so that all poles of the integrand $\Gamma(z) K\left((f(A)-m I)^{-z} ; x, y\right) t^{-z}$ are to the left of the contour. We then shift the contour across the poles of 
the integrand. These are due to the Gamma function and are located at $z=0,-1,-2, \ldots$ We find by Cauchy's theorem that

$$
K\left(e^{-(f(A)-m I) t} ; x, y\right)=\sum_{k=0}^{N} \frac{(-1)^{k}}{k !} K\left((f(A)-m I)^{k} ; x, y\right) t^{k}+R_{N}(x, y)
$$

with remainder term

$$
R_{N}(x, y)=\frac{1}{2 \pi i} \int_{c_{N}-i \infty}^{c_{N}+i \infty} \Gamma(z) K\left((f(A)-m I)^{-z} ; x, y\right) t^{-z} d z
$$

where the contour $c_{N}$ satisfies $-(N+1)<c_{N}<-N$ so that exactly $N$ poles lie to its right. Since we assumed $x \in K_{1}$ and $y \in K_{2}$ with $K_{1}$ and $K_{2}$ disjoint the zero-order term vanishes: the corresponding (distributional) Schwartz kernel is concentrated in the diagonal $x=y$ and zero elsewhere.

To estimate the remainder $R_{N}(x, y)$ note that since $(f(A)-m I)$ belongs to $\Psi D O_{c l}^{-2 z}(M)$, its kernel $K\left((f(A)-m I)^{-z} ; x, y\right)$ is the Fourier transform of a symbol of order $-2 \operatorname{Re} z$, cf. $[1,31]$. This means that for Re $z$ sufficiently large, the absolute value $\left|K\left((f(A)-m I)^{-z} ; x, y\right)\right|$ is bounded above independently of $z$ and of $x, y$. The bound on the Gamma function (4.6) gives a bound of $R_{N}$ in the form $\left|R_{N}(x, y)\right| \leq C_{N} t^{-c_{N}}$. Since $-(N+1)<c_{N}<-N$ we have for $t<1$ that $t^{-c_{N}}<t^{N}$, so

$$
\left|K\left(e^{-(f(A)-m I) t} ; x, y\right)-\sum_{k=1}^{N} \frac{(-1)^{k}}{k !} K\left((f(A)-m I)^{k} ; x, y\right) t^{k}\right| \leq C_{N} t^{N}
$$

which proves the asymptotic property in $C\left(K_{1} \times K_{2}\right)$.

Also, $K\left(e^{-(f(A)-m I) t} ; x, y\right)=e^{m t} K\left(e^{-f(A) t} ; x, y\right)$ from the series representation of $K\left(e^{-(f(A)-m I) t} ; x, y\right)$ in terms of eigenfunctions (4.3).

Remark 4.9. If $f(A)-m I$ were a local operator, then the argument of Remark 4.6 shows that the kernels of the integer powers $(f(A)-m I)^{k}$ would vanish and the heat kernel of $f(A)$ would decay faster than any polynomial in $t$ as $t \rightarrow 0$. So the nonlocality of $f(A)$ is a key part in this argument.

We are now ready to prove the heat kernel asymptotics of $f(A)$.

Proof of Theorem 3.5. We compute the lowest-order term in (3.3) in powers of the geodesic distance $d(x, y)$. Recall from Proposition 4.5 (ii) that we had

$$
f(A)-m I=a_{0} A^{\alpha}+a_{1} A^{\alpha-1}+R_{2 \alpha-4}
$$

with remainder term $R_{2 \alpha-4} \in \Psi D O^{2 \alpha-4}(M)$. We will estimate the summands on the right and side of (4.9) in terms of powers of $d(x, y)$.

1. The third summand can be bounded using a standard result on the behaviour of Schwartz kernels of pseudodifferential operators. By Proposition 2.2 in Chapter 7 of [34] we have that for $Q \in \Psi D O^{r}\left(\mathbb{R}^{n}\right)$, its Schwartz kernel satisfies the estimate

$$
|K(Q ; x, y)| \leq C|x-y|^{-n-r}
$$

provided $r>-n$. Now fix $y \in M$ and choose normal coordinates around $y$. If $x$ is in this coordinate patch, then the above estimate holds with $|x-y|$ 
replaced by the geodesic distance $d(x, y)$. Using (4.10) we obtain that the kernel of the remainder term $R_{2 \alpha-4}$ in (4.9) is bounded as $\left|K\left(R_{2 \alpha-4} ; x, y\right)\right| \leq$ $C d(x, y)^{-n-(2 \alpha-4)}$ for some constant $C$.

2. From Theorem 3.4 (i) we obtain with $n>4-2 \alpha$ and $z=-\alpha$ that

$$
\begin{aligned}
\Gamma(-\alpha) K\left(A^{\alpha} ; x, y\right) \\
=\frac{H_{0}(x, y)}{(2 \pi)^{n / 2}} \frac{\Gamma\left(\frac{n}{2}+\alpha\right)}{\left(\frac{1}{4} d(x, y)^{2}\right)^{n / 2+\alpha}}+\frac{H_{1}(x, y)}{(2 \pi)^{n / 2}} \frac{\Gamma\left(\frac{n}{2}+\alpha-1\right)}{\left(\frac{1}{4} d(x, y)^{2}\right)^{n / 2+\alpha-1}} \\
\quad+O\left(d(x, y)^{-n-2 \alpha+4}\right)
\end{aligned}
$$

and

$$
\begin{aligned}
& \Gamma(-(\alpha-1)) K\left(A^{\alpha-1} ; x, y\right) \\
& \quad=\frac{H_{0}(x, y)}{(2 \pi)^{n / 2}} \frac{\Gamma\left(\frac{n}{2}+\alpha-1\right)}{\left(\frac{1}{4} d(x, y)^{2}\right)^{n / 2+\alpha-1}}+O\left(d(x, y)^{-n-2 \alpha+4}\right),
\end{aligned}
$$

where $H_{0}$ and $H_{1}$ are from (3.1).

3. Overall we find

$$
\begin{aligned}
K( & f(A)-m I ; x, y) \\
= & a_{0} K\left(A^{\alpha} ; x, y\right)+a_{1} K\left(A^{\alpha-1} ; x, y\right)+O\left(d(x, y)^{-n-2 \alpha+4}\right) \\
= & \frac{a_{0}}{\Gamma(-\alpha)}\left[\frac{H_{0}(x, y)}{(2 \pi)^{n / 2}} \frac{\Gamma\left(\frac{n}{2}+\alpha\right)}{\left(\frac{1}{4} d(x, y)^{2}\right)^{n / 2+\alpha}}+\frac{H_{1}(x, y)}{(2 \pi)^{n / 2}} \frac{\Gamma\left(\frac{n}{2}-1+\alpha\right)}{\left(\frac{1}{4} d(x, y)^{2}\right)^{n / 2+\alpha-1}}\right] \\
& +\frac{a_{1}}{\Gamma(-(\alpha-1))} \frac{H_{0}(x, y)}{(2 \pi)^{n / 2}} \frac{\Gamma\left(\frac{n}{2}+\alpha-1\right)}{\left(\frac{1}{4} d(x, y)^{2}\right)^{n / 2+\alpha-1}}+O\left(d(x, y)^{-n-2 \alpha+4}\right) \\
= & \frac{a_{0}}{\Gamma(-\alpha)} \frac{H_{0}(x, y)}{(2 \pi)^{n / 2}} \frac{\Gamma\left(\frac{n}{2}+\alpha\right)}{\left(\frac{1}{4} d(x, y)^{2}\right)^{n / 2+\alpha}} \\
& +\left[\frac{a_{0}}{\Gamma(-\alpha)} \frac{H_{1}(x, y)}{(2 \pi)^{n / 2}}+\frac{a_{1}}{\Gamma(1-\alpha)} \frac{H_{0}(x, y)}{(2 \pi)^{n / 2}}\right] \frac{\Gamma\left(\frac{n}{2}+\alpha-1\right)}{\left(\frac{1}{4} d(x, y)^{2}\right)^{n / 2+\alpha-1}} \\
& +O\left(d(x, y)^{-n-2 \alpha+4}\right),
\end{aligned}
$$

whence equation (3.4) follows using the identity $\Gamma(-\alpha)=\Gamma(1-\alpha) /(-\alpha)$. This finishes the proof of assertion (ii) Theorem 3.5.

Remark 4.10. Note that that the dimensional restriction $n>4-2 \alpha$ in Theorem 3.5 is due to the bound (4.10). More generally, if one extended the sum in (4.9) up to order $A^{\alpha-(k-1)}$, the bound of the corresponding error term would hold for $n>2 k-2 \alpha$ indicating a trade-off between the order of the approximation and the dimension of the manifold $M$.

Aronson-type upper bounds of $K\left(e^{-t f(A)} ; x, y\right)$ are now easy to obtain.

Proof of Corollary 3.7. We recall the upper bounds of Theorem 1 of [16]. In our notation, their equation $(*)$ reads

$$
K\left(e^{-t(f(A)-m I)} ; x, y\right) \leq C_{1} t e^{-c t}\left(d(x, y)+t^{1 / 2 \alpha}\right)^{-n-2 \alpha}
$$


where $C_{1}$ is a constant and $c$ is less than the infimum of the spectrum of $f(A)-m I$. The infimum of the spectrum of $f(A)-m I$ is greater than 0 since $A$ has positive real spectrum, $f$ takes only nonnegative values and $m<0$. So we can choose $c=0$ yielding

$$
K\left(e^{-t(f(A)-m I)} ; x, y\right) \leq C_{1} t\left(d(x, y)+t^{1 / 2 \alpha}\right)^{-n-2 \alpha} .
$$

The upper bound in terms of $H(x, y)$ follows from Theorem 3.5 (ii) as

$$
K\left(e^{-t(f(A)-m I)} ; x, y\right) \leq C_{2} t H(x, y)
$$

using the compactness of $M$ to bound the error term of order $d(x, y)^{-n-2 \alpha+4}$ in terms of $d(x, y)^{-n-2 \alpha+2}$, the highest power of $d$ in $H$. Now set $C=$ $\max \left\{C_{1}, C_{2}\right\}$ to conclude.

\section{Subordinate Brownian Motion on a Closed Manifold}

Let $M$ be a closed manifold of dimension $n$ with Laplace-Beltrami operator $\Delta$. Then $\frac{1}{2} \Delta$ is the generator of the standard Brownian motion $B=\left(B_{t}\right)_{t \geq 0}$ on $M$, cf. [21].

We refer to the monograph [29] for details of subordinate stochastic processes. Let $\left(X_{t}\right)_{t \geq 0}$ be a subordinator on $[0, \infty)$ independent of $B$, i.e. an increasing Lévy process with values in $[0, \infty)$ and $X_{0}=0$ almost surely. We can characterise the distribution of $X_{t}$ by its generating function

$$
\mathbb{E}\left(e^{-\lambda X_{t}}\right)=e^{-t f(\lambda)},
$$

for $t \geq 0$ and $\lambda>0$ where $f$ is the the Laplace exponent of $X_{t}$. Here, $f$ has the additional property that it is a Bernstein function. This means that $f$ is smooth, $f(\lambda) \geq 0$ and $(-1)^{k-1} f^{(k)}(\lambda) \geq 0$ for $k \in \mathbb{N}$.

The Bernstein function is defined on the entire spectrum of $\frac{1}{2} \Delta$ so that this is a particularly straightforward case of functional calculus related to the subordination of semigroups.

Proposition 5.1. ([3]; [22], Chapter 4.3) The generator of the process $\left(B_{X_{t}}\right)_{t \geq 0}$ is $f\left(\frac{1}{2} \Delta\right)$ as defined by the spectral theorem.

The heat kernel on $M$ has an asymptotic expansion as $t \rightarrow 0$ of the form (cf. Theorem 2.30 of [5] or Theorem 5.1.1 of [21])

$$
K\left(e^{-\frac{1}{2} \Delta t} ; x, y\right) \sim\left(\frac{1}{2 \pi t}\right)^{n / 2} e^{-d(x, y)^{2} / 2 t} \sum_{m=0}^{\infty} H_{m}(x, y) t^{m},
$$

which is valid if $x$ and $y$ are not within each other's cut locus. Here, $d(x, y)$ is the geodesic distance of $x, y \in M$. The smooth functions $H_{m}$ have the property that we obtain the classical heat invariants of $M$ (volume, total scalar curvature, ...) as integrals along the diagonal $\int_{M} H_{m}(x, x) d x$.

To obtain the heat kernel asymptotics of $f\left(\frac{1}{2} \Delta\right)$ we would like to use the framework of Sect. 3. This, however, hinges on the existence of the complex powers of $A=\frac{1}{2} \Delta$. Since the lowest eigenvalue of this operator is zero we cannot apply Theorem 3.5 directly. Instead, we consider the operator $A_{\epsilon}=$ 
$\frac{1}{2} \Delta+\epsilon P$, where $P$ is the projection onto the finite-dimensional kernel of $\Delta$ and $\epsilon>0$ is small. This perturbation has the following effects

(i) The symbols of $A$ and $A_{\epsilon}$ agree so that on the scale of Sobolev spaces we only adjust by an operator of order $-\infty$.

(ii) The lowest eigenvalue $\mu_{1}$ of $A_{\epsilon}$ is positive, namely it is given by $\mu_{1}=\epsilon$.

(iii) We can form the complex powers of $A_{\epsilon}$ in the algebra of classical pseudodifferential operators.

(iv) Explicitly, the heat kernel of $A$ and $A_{\epsilon}$ are related by (for simplicity we assume a one-dimensional null space of $A$ with basis vector $\varphi_{0}$ )

$$
K\left(e^{-A_{\epsilon} t} ; x, y\right)=K\left(e^{-A t} ; x, y\right)+\left(e^{-\epsilon t}-1\right) \varphi_{0}(x) \varphi_{0}(y)
$$

so that the heat kernel asymptotics of $k_{A}$ and $k_{A_{\epsilon}}$ are identical in the orders $t^{-n / 2}, \ldots, t^{-1}$ and $t^{0}$. This is sufficient for our purposes.

We can thus apply the approach with $A_{\epsilon}$ and in the end let $\epsilon \rightarrow 0$. Note that due to $A$ being one-half the Laplacian, the asymptotics of $A^{-z}$ are in powers of $\frac{1}{2} d(x, y)^{2}$ as opposed to $\frac{1}{4} d(x, y)^{2}$.

Theorem 5.2. Assume that the dimension $n$ of the closed manifold $M$ satisfies $n>4-2 \alpha$. Suppose that the Laplace exponent $f$ satisfies Hypothesis 3.3. Let for $x, y \in M$ such that $x$ and $y$ are not in each other's cut locus. Then the short-time asymptotics of the transition density of the subordinate Brownian motion are given by

$$
K\left(e^{-f\left(\frac{1}{2} \Delta\right) t} ; x, y\right) \sim t e^{-m t}\left[H(x, y)+O\left(d(x, y)^{-n-2 \alpha+4}\right)\right]
$$

as $t \rightarrow 0^{+}$and

$$
\begin{aligned}
H(x, y)= & \frac{\Gamma\left(\frac{n}{2}+\alpha\right) 2^{n / 2+\alpha}}{\Gamma(1-\alpha)(2 \pi)^{n / 2}} \cdot \frac{\alpha a_{0} H_{0}(x, y)}{d(x, y)^{n+2 \alpha}} \\
& +\frac{\Gamma\left(\frac{n}{2}+\alpha-1\right) 2^{n / 2+\alpha-1}}{\Gamma(1-\alpha)(2 \pi)^{n / 2}} \cdot \frac{\alpha a_{0} H_{1}(x, y)-a_{1} H_{0}(x, y)}{d(x, y)^{n+2 \alpha-2}}
\end{aligned}
$$

where $H_{0}, H_{1}$ are from the heat expansion (5.2).

One can view $\alpha$ as a probabilistic parameter and the dimension $n$, the coefficient functions $H_{0}, H_{1}$ and the geodesic distance $d$ as geometric objects. Thus, the above expansion mixes the geometry of the manifold and probabilistic information.

By Corollary 3.7, we find an upper bound for the heat kernel

$$
K\left(e^{-f\left(\frac{1}{2} \Delta\right) t} ; x, y\right) \leq C t e^{-m t}\left(\left(d(x, y)+t^{1 / 2 \alpha}\right)^{-n-2 \alpha} \wedge H(x, y)\right)
$$

for $t \in(0,1)$ and any $x, y \in M$.

In the case of the relativistic $\alpha$-stable processes, i.e. $f(\lambda)=(1+\lambda)^{\alpha}-1$, we can compare this to [11]. Although the authors consider processes living on a bounded domain $D \subset \mathbb{R}^{n}$, a comparison of the heat kernel bounds is nonetheless instructive. Using sophisticated probabilistic methods the authors obtain in their Theorem 1.1 the upper bound

$$
K\left(e^{-f(\Delta) t} ; x, y\right) \leq C\left(1 \wedge \frac{\delta(x)^{\alpha}}{\sqrt{t}}\right)\left(1 \wedge \frac{\delta(y)^{\alpha}}{\sqrt{t}}\right)\left(t^{-n / 2 \alpha} \wedge \frac{t \varphi\left(\frac{|x-y|}{16}\right)}{|x-y|^{n+2 \alpha}}\right)
$$


for a constant $C$ where $\delta(\cdot)$ measures the distance to the boundary and $\varphi(r)=$ $e^{-r}\left(1+r^{(n+2 \alpha-1) / 2}\right)$. (Since [11] is set on domains in $\mathbb{R}^{n}$, the generator of Brownian motion is $\Delta$ and not $\frac{1}{2} \Delta$ as is the case on closed manifolds.)

This seems to suggest that bounds of this type hold for a larger class of subordinate Brownian motion and not just for the relativistic $\alpha$-stable processes.

\section{Acknowledgements}

The author thanks the anonymous referee for several helpful suggestions.

Open Access. This article is distributed under the terms of the Creative Commons Attribution 4.0 International License (http://creativecommons.org/licenses/ by/4.0/), which permits unrestricted use, distribution, and reproduction in any medium, provided you give appropriate credit to the original author(s) and the source, provide a link to the Creative Commons license, and indicate if changes were made.

\section{References}

[1] Agranovich, M.S.: Elliptic operators on closed manifolds. In: Egorov, Yu.V., Shubin, M.A. (eds.) Partial Differential Equations VI, Volume 63 of Encyclopaedia of Mathamatics and Science, pp. 1-130. Springer, Berlin (1994)

[2] Baldus, F.: Application of the Weyl-Hörmander calculus to generators of Feller semigroups. Math. Nachr. 252(1), 3-23 (2003)

[3] Bañuelos, R., Baudoin, F.: Trace Asymptotics for Subordinate Semigroups. arXiv:1308.4944 (2013)

[4] Arous, G.B.: Développement asymptotique du noyau de la chaleur hypoelliptique hors du cut-locus. Ann. Sci. Éc. Norm. Supér 21(3), 307-331 (1988)

[5] Berline, N., Getzler, E., Vergne, M.: Heat Kernels and Dirac Operators. Grundlehren Math, vol. 298. Wiss. Springer, Berlin (1992)

[6] Bleistein, N., Handelsman, R.A.: Asymptotic Expansions of Integrals. Holt, Rinehart and Winston, New York (1975)

[7] Bochner, S.: Harmonic Analysis and the Theory of Probability. University of California Press, Berkeley (1955)

[8] Bogdan, K., Grzywny, T., Ryznar, M.: Heat kernel estimates for the fractional laplacian with dirichlet conditions. Ann. Prob. 38(5), 1901-1923 (2010)

[9] Chen, Z.-Q.: Symmetric jump processes and their heat kernel estimates. Sci. China (Ser. A) 52(7), 1423-1445 (2009)

[10] Chen, Z.-Q., Kim, P., Song, R.: Heat kernel estimates for the Dirichlet fractional Laplacian. J. Eur. Math. Soc. 12(5), 1307-1329 (2010)

[11] Chen, Z.-Q., Kim, P., Song, R.: Sharp heat kernel estimates for relativistic stable processes in open sets. Ann. Probab. 40(1), 213-244 (2012)

[12] Davies, E.B.: Heat Kernels and Spectral Theory. Cambridge Tracts in Math, vol. 92. Cambridge University Press, Cambridge (1990)

[13] Dunau, J.: Fonctions d'un opérateur elliptique sur une variété compacte. J. Math. Pures Appl. 56(4), 367-391 (1977) 
[14] Fahrenwaldt, M.A.: Heat trace asymptotics of subordinated Brownian motion on Euclidean space. Potential Anal. 44, 331-354 (2016)

[15] Fahrenwaldt, M.A.: Spectral functions of subordinate brownian motion on closed manifolds. J. Lond. Math. Soc. 93(2), 703-720 (2016)

[16] Gimperlein, H., Grubb, G.: Heat kernel estimates for pseudodifferential operators, fractional Laplacians and Dirichlet-to-Neumann operators. J. Evol. Equ. 14(1), 49-83 (2014)

[17] Grigor'yan, A.: Estimates of heat kernels on Riemannian manifolds. In: E.B. Davies and Yu. Safarov (eds.) Spectral Theory and Geometry. ICMS Instructional Conference, Edinburgh 1998, volume 273 of London Mathamatical Society Lecture Note Series, pp. 140-225. Cambridge, Cambridge University Press (1999)

[18] Grigor'yan, A.: Heat Kernel and Analysis on Manifolds. AMS/IP Studies in Advanced Mathematics, vol. 47. American Mathematical Society, Providence (2012)

[19] Hassell, A., Vasy, A.: Symbolic functional calculus and $n$-body resolvent estimates. J. Funct. Anal. 173(2), 257-283 (2000)

[20] Hoh, W.: A symbolic calculus for pseudo differential operators generating Feller semigroups. Osaka J. Math 35(4), 789-820 (1998)

[21] Hsu, E.P.: Stochastic analysis on manifolds. Grad, vol. 38. Stud. Math. American Mathematical Society, Providence (2002)

[22] Jacob, N.: Pseudo Differential Operators \& Markov Processes: Fourier Analysis and Semigroups, vol. 1. Imperial College Press, London (2001)

[23] Jacob, N., Knopova, V., Landwehr, S., Schilling, R.L.: A geometric interpretation of the transition density of a symmetric Lévy process. Sci. China Math. 55(6), 1099-1126 (2012)

[24] Phillips, R.S.: On the generation of semigroups of linear operators. Pac. J. Math. 2(3), 343-369 (1952)

[25] Ponge, R.: Heisenberg calculus and spectral theory of hypoelliptic operators on Heisenberg manifolds. Mem, vol. 194. American Mathematical Society, Providence (2008)

[26] Remmert, R.: Classical Topics in Complex Function Theory. Grad, vol. 172. Texts in Math. Springer, New York (1997)

[27] Schilling, R.L.: On the domain of the generator of a subordinate semigroup. In: Potential theory-ICPT 94 (Kouty, 1994), pp. 449-462. de Gruyter, Berlin (1996)

[28] Schilling, R.L.: Subordination in the sense of Bochner and a related functional calculus. J. Austral. Math. Soc. (Ser. A) 64(03), 368-396 (1998)

[29] Schilling, R.L., Song, R., Vondracek, Z.: Bernstein Functions: Theory and Applications. Studies in Mathematics, vol. 37, 2nd edn. de Gruyter, Berlin (2012)

[30] Scott, S.: Traces and Determinants of Pseudodifferential Operators. Oxford Math. Monogr. Oxford University Press, Oxford (2010)

[31] Seeley, R.T.: Complex powers of an elliptic operator. Proc. Sympos. Pure Math. 10, 288-307 (1967)

[32] Shubin, M.A.: Pseudodifferential Operators and Spectral Theory, 2nd edn. Springer, Berlin (2001) 
[33] Strichartz, R.S.: A functional calculus for elliptic pseudo-differential operators. Am. J. Math. 94(3), 711-722 (1972)

[34] Taylor, M.E.: Partial Differential Equations II: Qualitative Studies of Linear Equations. Appl, vol. 116, 2nd edn. Mathamatical Science. Springer Science \& Business Media, New York (2013)

[35] Titchmarsh, E.C.: Introduction to the Theory of Fourier Integrals. Clarendon Press, Oxford (1948)

\author{
M. A. Fahrenwaldt $(\bowtie)$ \\ Department of Actuarial Mathematics and Statistics \\ Maxwell Institute for Mathematical Sciences \\ Heriot-Watt University \\ Edinburgh EH14 4AS \\ UK \\ e-mail: m.fahrenwaldt@hw.ac.uk
}

Received: July 29, 2016.

Revised: December 28, 2016. 